\title{
MODELING SOCIAL RISK AMPLIFICATION DURING RECALL CRISES
}

\author{
Yun Liu \\ Department of Management Science \\ Lancaster University \\ Bailrigg, Lancaster LA1 4YX, UK \\ y.liu30@lancaster.ac.uk
}

\author{
Jerry Busby \\ Department of Management Science \\ Lancaster University \\ Bailrigg, Lancaster LA1 4YX, UK \\ j.s.busby@lancaster.ac.uk
}

\author{
Bhakti Stephan Onggo \\ Department of Management Science \\ Lancaster University \\ Bailrigg, Lancaster LA1 4YX, UK \\ s.onggo@lancaster.ac.uk
}

\begin{abstract}
Public response to risk is socially shaped in a way that often severely over- or under-estimates expert risk assessments. One of the main theoretical tools to examine public risk responses of this kind is the social amplification of risk framework. An important finding is that social amplification is strongly influenced by the conduct of those managing a risk. This study models social risk amplification as a collective response to risks that are significant at a societal level but which materialize in a distributed way across a population. It uses the context of product contamination risks and product recalls to show how organizational decision making and the multiple roles of the media can be represented by the model. It shows how a model can be partially calibrated with a consumer survey, and shows which are the most important aspects of the model in shaping social risk amplification.
\end{abstract}

Keywords: social risk amplification, product recall, recreancy, media.

\section{INTRODUCTION}

Risk perception appears to hold a central position in the social and organizational agenda and is crucial for the understanding of social processing of uncertainty (Rogers 1997). It has long been accepted that public perceptions of risk are socially shaped (Scherer and Cho 2003, Muter et al. 2013). The judgments that people make about risks are more a question of social interaction and observation than of merely anticipated physical consequences (Mitchell 1995, McComas 2003). One of the main approaches for explaining public risk perception is the social amplification of risk framework (SARF) (Kasperson et al. 1988). The central idea of the framework is that risk events interact with psychological, social, and cultural processes in ways that can amplify or attenuate public response to risk. Behavioral reactions to risks may produce ripple effects that trigger another stage of risk amplification. SARF emphasizes the role of various amplification stations (e.g. individuals, social groups, media, and so on) in conveying risk signals and in the formation of risk perception.

Past empirical work on social risk amplification has been concerned with the correlations of structural variables in risk amplification rather than the mechanism that produces risk amplification. According to 
Kasperson et al. (1988), the core of social risk amplification is the response mechanism of society. And more importantly, there has been quite limited modeling of risk amplification to date. Examples seen in the literature have used both system dynamics (Burns and Slovic 2007, Busby and Onggo 2013) and agent-based models (Busby et al. 2016, Onggo et al. 2014) to explore the mechanisms of risk amplification. Their primary concern has been how social communication influences the formation of risk beliefs. This concern has meant that other factors shaping risk responses have received little attention. One is organizational misconduct, which is termed as 'recreancy' and defined as the belief that the producer has betrayed the public trust and fails to fulfill its obligations (Freudenburg 1993). Freudenburg (2003) has argued that recreancy is an important contributor to social risk amplification. This combines with direct experience. Direct experience serves as a source of information by providing feedback on the nature and controllability of hazardous events (Kasperson et al. 1988, Kasperson 2012). Rogers (1997) pointed out that direct experience can lead to learned perception and is an important basis of public risk estimates, although it may be very limited.

Our aim in this paper is to describe a study in which we attempt to model situations in which collective risk response integrates direct experience with social communication and perception of organizational misconduct. Such an investigation has received little emphasis and is carried out to provide a more complete and integrated understanding of social risk amplification, especially for those responsible for managing risk. The inherent heterogeneity of individual risk perceivers (Sjöberg 2000), the emergence of behavior through interactions of social actors (Binder et al. 2011, Smith et al. 2013), and the complex feedback loops linking risk perception with risk related behavior (Burns and Slovic 2007, Busby and Onggo 2013, Busby et al. 2016) point to using an agent-based model as a modeling medium. We develop such a model in the context of product contamination scandals such as the recent cases in China of contaminated milk products. Product recall is often a key element in risk communication of productrelated crises (Magno 2012). Recalls send ambiguous signals about the misconduct of the producer in question: they clearly indicate some kind of failure, and possibly negligence, in the producer's processes; but they also indicate some kind of concern with consumers' welfare. The timing and voluntariness of recalls is pivotal in determining not just how information about a potential danger is disseminated, but also how the organization's conduct is judged - and how strongly consumers will be motivated to boycott a product, switch to substitutes, seek compensation or engage in protest.

The remainder of this paper is structured as follows. Section 2 describes the development of a model. Section 3 shows the process of calibrating the model using empirical data from a public survey. Section 4 briefly discusses the sensitivity of peak risk amplification to independent variables. The paper then ends by discussion and conclusion.

\section{DEVELOPMENT OF THE MODEL}

As shown in the conceptual model in Figure 1, an individual agent's risk perceptions are shaped by two essential processes: 1) the discovery of a danger and the processing of information indicating the scale of this danger; and 2) the formation of a perception of recreancy or misconduct. The discovery process draws information from that agent's prior beliefs, from the beliefs of others that it interacts with, from any direct experience (for example illness following ingestion of a contaminated food product) and from a producer's recall. The recreancy process involves assessing the timing and voluntariness of the recall process. In any specific, real case there may be many more considerations that influence the recreancy process, but at a minimum it should involve timing and voluntariness. These influences then combine with communication from the media of any kind relevant to an agent. 


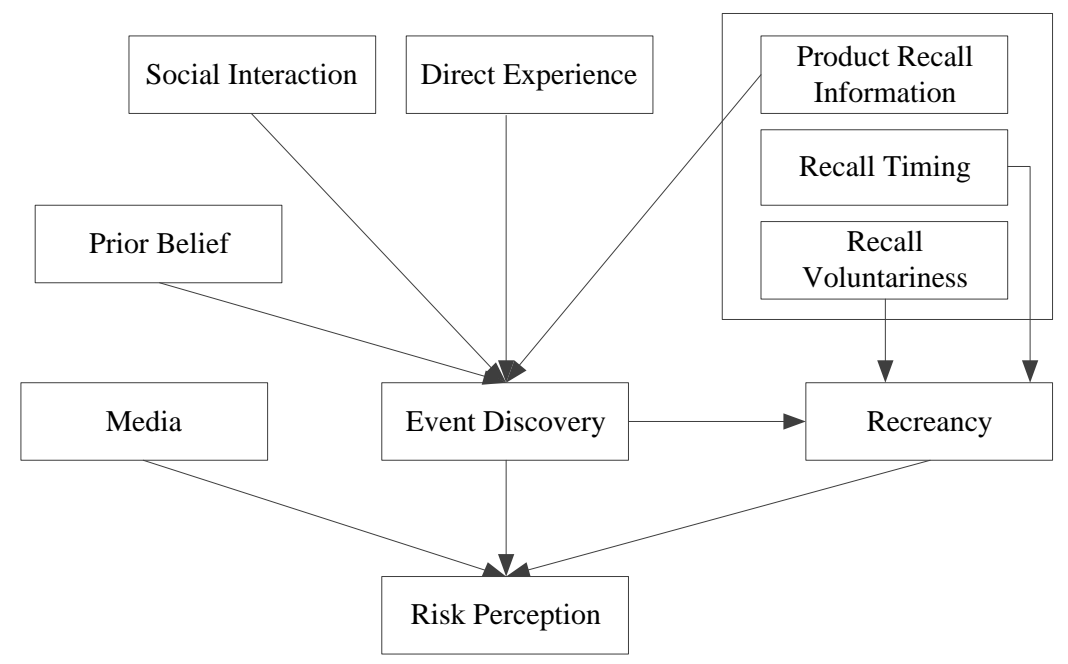

Figure 1: Conceptual model of a social agent developing a risk perception to a product recall.

\subsection{Basic Model}

In our basic model there are $N$ agents who interact with direct neighbors in a small-world network following Watts and Strogatz (1998). At the start each agent $i$ is endowed with a risk belief $b_{i}(t=0) \in[0, I)$, and an activated agent $i$ interacts with $K$ neighbors at a time. An agent $i$ updates this risk belief based on its prior belief and mean risk belief of neighbors, that is:

$$
b_{i}(t)=w_{1} b_{i}(t-1)+w_{2} \frac{1}{K} \sum_{n j=1}^{K} b_{n j}(t)
$$

The weights $w_{1}$ and $w_{2}$, as we described later, are calibrated from a survey.

\subsection{Adding Contamination}

A contamination level $C(t)$ expresses the probability that any activated agent will directly experience a harm. Contamination level is set as a constant in the model and its value is very small prior to and after the contamination incident and high during the incident:

$$
C(t)= \begin{cases}C_{\text {low }}(t) & 0 \leq t<T_{\text {start }}, t>T_{\text {end }} \\ C_{\text {high }}(t) & T_{\text {start }} \leq t \leq T_{\text {end }}\end{cases}
$$

Individuals are assumed to consume the product once, when active, and this product will be contaminated with a probability $C(t)$. Direct experience, $e_{i}(t) \in\{0,1\}$, is binary and added to the decision rule:

$$
b_{i}(t)=w_{1} b_{i}(t-1)+w_{2} \frac{1}{K} \sum_{n j=1}^{K} b_{n j}(t)+w_{3} e_{i}(t)
$$

where $w_{3}$ represents the weight of direct experience.

\subsection{Adding Product Recall}

There are two main aspects to the effect of a product recall on public perceptions: 1) it provides information that the product is defective, which combines with the three sources of information already described to determine public risk beliefs; 2) it increases or decreases the public's trust in the company depending on its timing, and whether it is voluntary. 
We assume a single producer agent. The producer issues a recall message during the time between contamination release and contamination termination, but does not withdraw the product. The recall is issued with a probability equal to the contamination risk $C(t)$ at any given time. This means the recall timing is random. There is likely to be some, small delay between the sudden contamination increase and the recall. After a recall announcement has been made, the recall, $r(t) \in\{0,1\}$, stays in force until the contamination level falls to its original, very low level, that is, $r(t)=0$ until $a(t)=1$, then $r(t)=1$ until $t=T_{\text {end }}$, and then $r(t)=0$. The public's risk belief decision rule now also incorporates recall information (simply a binary value):

$$
b_{i}(t)=w_{1} b_{i}(t-1)+w_{2} \frac{1}{K} \sum_{n j=1}^{K} b_{n j}(t)+w_{3} e_{i}(t)+w_{4} r(t)
$$

where $w_{4}$ is the weight of product recall information, and $w_{1}+w_{2}+w_{3}+w_{4}=1$.

\subsection{Adding Recreancy}

As an early, voluntary product recall indicates that a firm is acting in a socially responsible way, and a late, forced recall indicates the opposite, recreancy is influenced by the timing of the recall. It is also influenced by its voluntariness: if the producer is forced by the authorities to recall the products, recreancy will be higher than if not. So only if the recall is broadcast before the consumer believes the risk has increased and is voluntary, perceived recreancy will be low and this will reduce risk perceptions.

Each agent expresses a recreancy belief $R_{i}(t=0) \in[0, I)$ from the start. With no recall in force, the recreancy perceived by an agent will increase by some increment $D$, if and when its risk perception $b_{i}(t)$ increases above some threshold $B$, but stay unchanged otherwise. In this condition the agent has determined there is a risk and yet heard no recall. The updating rule for recreancy $R_{i}(t)$ is thus:

$$
R_{i}(t)= \begin{cases}R_{i}(t-1) & b_{i}(t-1)<B \& b_{i}(t)<B, 0 \leq t<t_{r=1} \& t>T_{\text {end }} \\ R_{i}(t-1)+D & b_{i}(t-1)<B \& b_{i}(t) \geq B, 0 \leq t<t_{r=1} \& t>T_{\text {end }}\end{cases}
$$

To make sure that recreancy is altered only once, we introduce another variable $h_{i}(t)$ to denote whether an agent has already heard the recall:

$$
h_{i}(t)= \begin{cases}0 & 0 \leq t<t_{r=1} \& t>T_{\text {end }} \\ 1 & t_{r=1} \leq t \leq T_{\text {end }}\end{cases}
$$

Also, there is a dummy variable, $v(t) \in\{0,1\}$, which denotes that the recall is voluntary $(v(t)=1)$ or involuntary $(v(t)=0)$. The associated decision rule is that if a recall is forced $(v(t)=0)$ and an agent hears the recall ( $h_{i}(t)=1$ ), then recreancy $R_{i}(t)$ is increased by some increment $E$, and that if a recall is made voluntarily $(v(t)=1)$ and an agent hears the recall $\left(h_{i}(t)=1\right)$, then recreancy $R_{i}(t)$ is reduced by the same amount:

$$
R_{i}(t)= \begin{cases}1 & v(t)=0 \& h_{i}(t)=1 \& R_{i}(t-1)+E>1 \\ R_{i}(t-1)+E & v(t)=0 \& h_{i}(t)=1 \& R_{i}(t-1)+E \leq 1 \\ 0 & v(t)=1 \& h_{i}(t)=1 \& R_{i}(t-1)<E \\ R_{i}(t-1)-E & v(t)=1 \& h_{i}(t)=1 \& R_{i}(t-1) \geq E\end{cases}
$$


where $t_{r=1} \leq t \leq T_{\text {end }}$.

We then combine the risk perception emerging from the discovery process outlined earlier (see equation (4)) with that shaped by recreancy in an extended decision rule:

$$
b_{i}(t)=w_{5}\left(w_{1} b_{i}(t-1)+w_{2} \frac{1}{K} \sum_{n j=1}^{K} b_{n j}(t)+w_{3} e_{i}(t)+w_{4} r(t)\right)+w_{6} R_{i}(t)
$$

where $w_{5}$ and $w_{6}$ are the weight given to 'event discovery' and 'recreancy', respectively.

\subsection{Adding Broadcast Media}

Onggo et al. (2014) have proposed three different roles that media can assume: it can lead public opinion and communicate an objective level of risk (role 1), it can follow public opinion by communicating the current, average public opinion (role 3), or it can be a mixed leader-follower (role 2). In our model the media agent becomes active at the start of contamination. Thus the media communication is:

$$
M(t)=\left\{\begin{array}{lll}
0 & 0 \leq t<T_{\text {start }} & \\
C(t) & t \geq T_{\text {start }} & \text { [role 1] } \\
\frac{1}{2}\left(C(t)+\frac{1}{N} \sum_{i=1}^{N} b_{i}(t-1)\right) & t \geq T_{\text {start }} & \text { [role 2] } \\
\frac{1}{N} \sum_{i=1}^{N} b_{i}(t-1) & t \geq T_{\text {start }} & \text { [role 3] }
\end{array}\right.
$$

We then integrate the effect of media communication to the decision rule in equation (8) to form the final decision rule in the model:

$$
b_{i}(t)=w_{5}\left(w_{1} b_{i}(t-1)+w_{2} \frac{1}{K} \sum_{n j=1}^{K} b_{n j}(t)+w_{3} e(t)+w_{4} r(t)\right)+w_{6} R_{i}(t)+w_{7} M(t)
$$

where $w_{5}, w_{6}$, and $w_{7}$ are the weight given to 'event discovery', 'recreancy', and the perception expressed in the 'media', $0<w_{5}<1,0<w_{6}<1,0<w_{7}<1$, and $w_{5}+w_{6}+w_{7}=1$.

\section{CALIBRATION SURVEY}

\subsection{Survey Design}

The aim of the survey is to provide a certain level of calibration for the agent-based model. It performs what is often referred to as 'micro-validation' (Moss and Edmonds 2005) by ensuring elements in the model correspond to empirically determined values. For this model, the micro-validation has two main elements: 1) assessing the information sources that people consult when forming risk perceptions; 2) assessing the relative importance they give to these sources in their decision rules. This produces empirical distributions from which the weights in the decision rules shown in Section 2 can be sampled in the agent model.

The survey was carried out in the context of liquid milk contamination. The questions were divided into three sections - the first finding out the demographic profile of the respondents, the second dealing with which other agents the respondents expect to get information from, the third looking at the relative importance of the different types of source. For the convenience of quantitative model calibration, all comparisons were made relative to the same baseline, i.e. social interaction, except for the comparison between recall timing and recall voluntariness. For each pair of comparisons, both forward comparison 
(e.g. when you form your risk perception, how much relative importance would you give to media communicated risk compared with other people's perceptions?) and reverse comparison (e.g. when you form your risk perception, how much relative importance would you give to other people's perceptions compared with media communicated risk?) were considered and randomly presented in the questionnaire in order to reduce cognitive biases that potential respondents may have.

The survey used a convenience sampling approach as the aim was to demonstrate the general approach, not model a specific population responding to a specific product crisis. But it was conducted in China, as product contamination crises have been particularly prominent as risk events in China - for example the contaminated milk scandals. The survey questionnaire was translated into Chinese before distribution, and the survey link was posted onto community websites (e.g. Guokr.com, Douban.com), academic forums (e.g. muchong.com, bbs.pinggu.org), and a chat application (e.g. WeChat) to collect responses.

\subsection{Survey Results}

Overall, 321 responses including 280 complete ones were received. In general, the demographic characteristics of the survey respondents were consistent with the China census data in terms of gender but differed in aspects of age, education, and income. The responses were mainly collected from community websites and academic forums that are popular for young and educated people, resulting in the fact that older and non-educated people are less likely to be included in the sample.

The results of the survey showed that respondents gave more importance to their own beliefs, direct experience, product recall information, and media coverage than perceptions from their social neighbors and that they weighted neighbors' perceptions more heavily than their trust in the producer. These results were not analyzed formally as the purpose of the survey was to produce sampling distributions for the model weights, as will now be described.

\subsection{Model Calibration}

Figure 2 briefly describes the process by which the model was calibrated using data drawn from the survey as follows: (1) $Q_{7}$ : ratio of prior belief to others' perceptions, (2) $Q_{8}$ : ratio of direct experience to others' perceptions, (3) $Q_{9}$ : ratio of recall notice to others' perceptions, (4) $Q_{10}$ : ratio of recall timing to recall voluntariness, (5) $Q_{11}$ : ratio of trust in the producer to others' perceptions, (6) $Q_{12}$ : ratio of media communication to others' perceptions.

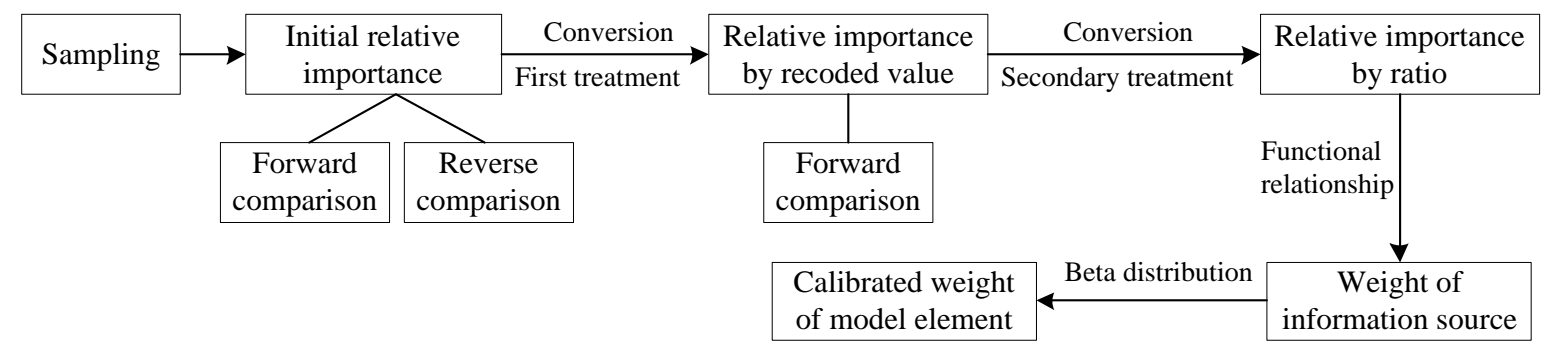

Figure 2: Model calibration process by survey data.

Based on the relationships between weights and questionnaire items, the weights that each respondent gave to different information sources were as below:

$$
\begin{aligned}
& w_{1}=\frac{Q_{7}}{1+Q_{7}+Q_{8}+Q_{9}}, w_{2}=\frac{1}{1+Q_{7}+Q_{8}+Q_{9}}, w_{3}=\frac{Q_{8}}{1+Q_{7}+Q_{8}+Q_{9}}, \\
& w_{4}=\frac{Q_{9}}{1+Q_{7}+Q_{8}+Q_{9}}, w_{5}=\frac{1+Q_{7}+Q_{8}+Q_{9}}{1+Q_{7}+Q_{8}+Q_{9}+Q_{11}+Q_{12}},
\end{aligned}
$$




$$
w_{6}=\frac{Q_{11}}{1+Q_{7}+Q_{8}+Q_{9}+Q_{11}+Q_{12}}, w_{7}=\frac{Q_{12}}{1+Q_{7}+Q_{8}+Q_{9}+Q_{11}+Q_{12}} .
$$

The dataset obtained for each weight was fitted with a beta distribution, and these were then used as sampling distributions for the agents in the model, such that all agents follow the same updating rule but each agent adopted a unique set of parameter values sampled from these distributions. It is important to note, however, this represents only a partial calibration of the model, as there are model parameters such as the objective risk level, the threshold in risk perception, and the recreancy variation which are not examined by the survey. Also, each agent is endowed with a threshold in risk perception sampled from $[0, S)$ and a variation in recreancy threshold sampled from $[0, H)$ according to a uniform distribution. Further empirical work will be needed to calibrate these values. It is also important to recognize that parameters like the objective risk level are specific to every case being modeled. And in practice it may be hard to know what the objective risk level is, since expert risk assessments are often in disagreement.

\subsection{Simulation Results}

The simulation experiments are conducted using Repast Simphony. The model runs over a series of 20,000 periods with 1,000 public agents, a producer agent, and a media agent. Table 1 lists the input parameters and values used in the simulation. Figure 3 presents the traces of agent risk perception in a single run. Black lines represent mean public risk belief and grey lines standard deviation. Dotted lines denote the case where the media acts as an objective leader, dashed lines a mixed leader-follower, and solid lines a public follower. And the vertical line signifies recall announcement and the broken line contamination level. The results show that risk amplification - that is, a collective perception that exceeds the objective risk level - occurs regardless of the role that media assumes and of the voluntariness of recall. Compared with a voluntary product recall, an involuntary recall leads to a higher level of risk perception during the crisis as well as a higher residue of concern after the crisis. The objectivity of media coverage appears to be negatively related to risk amplification: a media that simply follows public opinion is associated more strongly with exaggerated risk perceptions than an objective one. It is significant that when the crisis finishes, and, the contamination has fallen to its original level, when the media is a public follower the public risk perception remains very high - it is not corrected by the reduction in objective risk. The indication is that the recall process and the role of media are important for risk amplification not just in the course of a crisis but after its resolution.
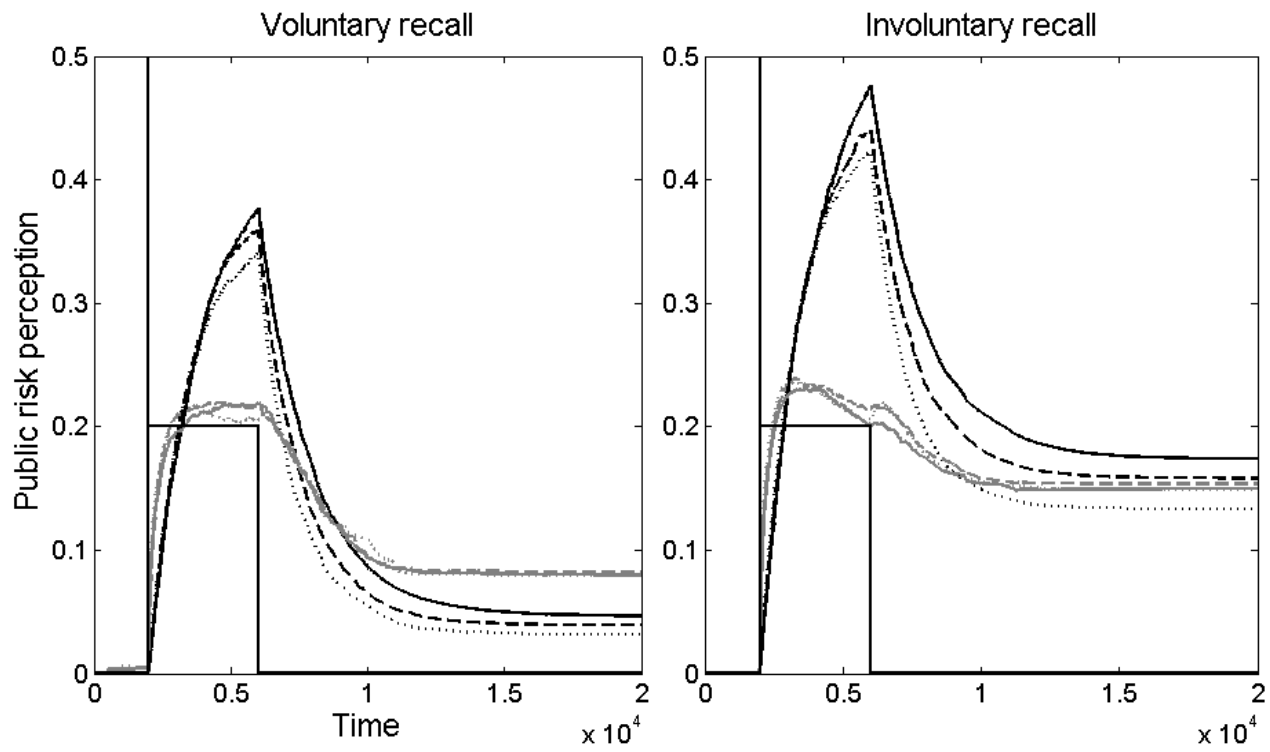

Figure 3: Simulation results in a single run. 
Liu, Busby, and Onggo

Table 1: Input parameters and values used in the simulation.

\begin{tabular}{|c|c|c|}
\hline Input Parameter & Default Value & Description \\
\hline Maximum initial condition $I$ & $10^{-4}$ & Defines initial risk and recreancy belief \\
\hline Number of neighbors $K$ & 4 & Number of neighbors in initial lattice \\
\hline Rewiring probability $p$ & 0.5 & Probability of reconnecting a lattice edge \\
\hline Low contamination level $C_{\text {low }}(t)$ & $10^{-4}$ & Level before and after crisis \\
\hline High contamination level $C_{\text {high }}(t)$ & 0.2 & Level during the crisis \\
\hline Contamination start period $T_{\text {start }}$ & 2000 & Time when the crisis starts \\
\hline Contamination end period $T_{\text {end }}$ & 5999 & Time when the crisis ends \\
\hline Recall voluntariness $v(t)$ & $\{0,1\}$ & Whether recall is voluntary or involuntary \\
\hline Maximum perception threshold $S$ & 1 & Defines when a recall increases recreancy \\
\hline Maximum of recreancy variation $H$ & 1 & Maximum by which recreancy can change \\
\hline
\end{tabular}

\section{SENSITIVITY ANALYSIS}

\subsection{Method of Sensitivity Analysis}

Sensitivity analysis in this study evaluates the main effect linking each of the model input parameters with defined outcome variables. Eight parameters are considered significant for defining the global uncertainty in model outputs. They are maximum initial condition $I$, number of neighbors $K$, rewiring probability $p$, low contamination level $C_{\text {low }}(t)$, high contamination level $C_{\text {high }}(t)$, contamination end period $T_{\text {end }}$, maximum of risk perception threshold $S$, and maximum of recreancy variation $H$. Given the limited space, we deal with only one outcome variable, peak risk amplification $\mu_{p}$ defined as the ratio between peak risk perception and objective risk level:

$$
\mu_{p}=\frac{1}{S} \sum_{j=1}^{S}\left(\max \left(\frac{1}{T} \sum_{t=1}^{T} \frac{1}{N} \sum_{i=1}^{N} b_{i}(t)\right) / C_{\text {high }}(t)\right)
$$

where $S, N$, and $T$ signify number of model runs, number of agents, and number of periods in a single run, and $C_{\text {high }}(t)$ is the contamination level during the crisis.

The approach used for sensitivity analysis is one-at-a-time (OAT). It examines the effect of one parameter at a time with all other parameters left at base values shown in Table 1. Each parameter is sampled 200 times uniformly from a specified range based on a subjective choice: $I:[0,1] ; K:[2,50] ; p:[0,1]$; $C_{\text {low }}(t):\left[10^{-6}, 10^{-3}\right] ; C_{\text {high }}(t):\left[2 \times 10^{-4}, 1\right] ; T_{\text {end }}:\left[3 \times 10^{3}, 16 \times 10^{3}\right] ; S:[0,1] ; H:[0,1]$. For each sample the model is run 100 times for the duration of 20,000 periods with 1,000 agents in a small-world network.

\subsection{Results of Sensitivity Analysis}

Both product moment correlation coefficients and scatter plots are used to depict the results. Correlation coefficients demonstrate that peak risk amplification is sensitive to maximum initial condition $I$, 
maximum of recreancy variation $H$ (in the case of involuntary recall), high contamination level $C_{\text {high }}(t)$, and contamination end period $T_{\text {end }}$. It is insensitive to number of neighbors $K$ and rewiring probability $p$, which indicates that the topology of the social network has no impact on social risk amplification. Besides, no dependence is found on low contamination level $C_{\text {low }}(t)$ and maximum of risk perception threshold $S$.

The following figures show visually the non-linear relationship between independent variables and peak risk amplification. As shown in Figure 4, in the context of voluntary recall, peak risk amplification increases with maximum of initial condition (which effectively is the maximum initial risk belief in the population). Peak risk amplification increases more rapidly when the initial condition exceeds a certain value, because at this point the exogenous peak starts occurring at the start of the model. This indicates that the collective risk responses are affected by a society's status quo. For high contamination level and contamination end period, the results with voluntary recall and with involuntary recall are qualitatively similar, so only one case is presented for illustration. Figure 5 illustrates that risk attenuation rather than risk amplification occurs when the objective risk level is beyond a certain value. As it can be seen from Figure 6, peak risk amplification increases with the duration of contamination to a certain level but goes no further. These suggest that peak risk amplification is sensitive to contamination in terms of both its magnitude and length and that the degree of sensitivity depends on the values of these two variables.

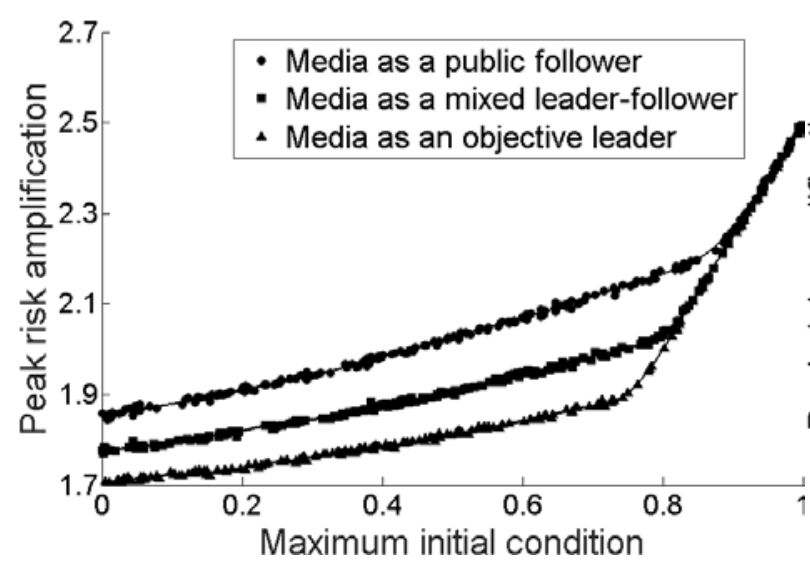

Figure 4: Maximum initial condition.

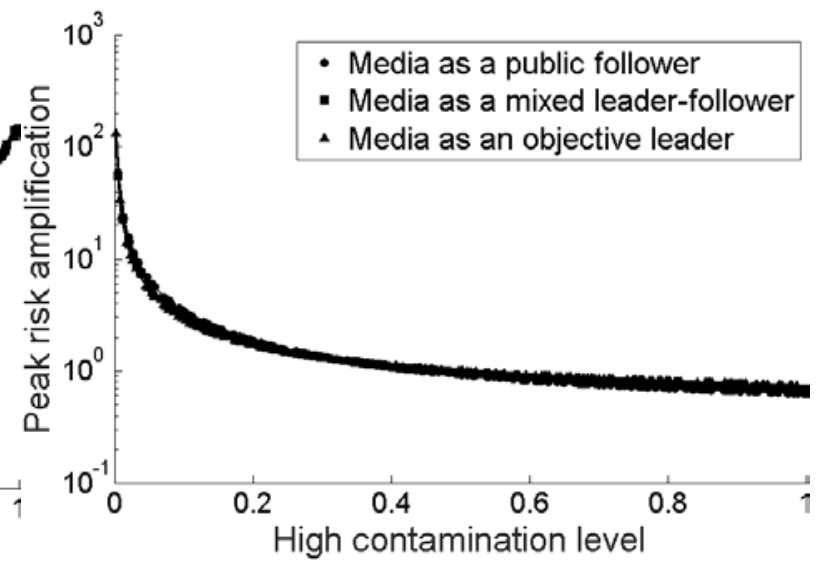

Figure 5: High contamination level.

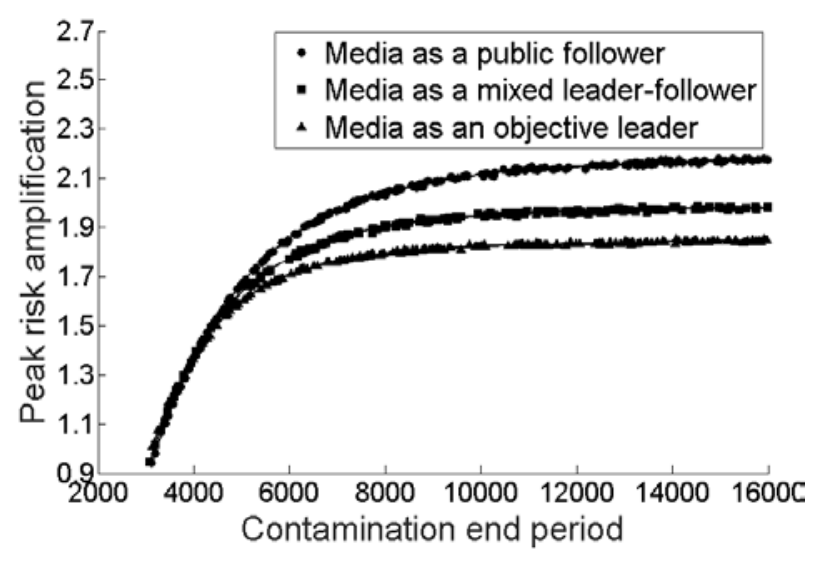

Figure 6: Contamination end period.

\section{DISCUSSION AND CONCLUSION}

This study uses an agent-based approach to model and simulate social risk amplification in a way in which SARF cannot be modeled directly. The systematic description of social experience of risk by 
SARF is very general. And there are some ambiguities inherent in SARF. For example, Rip (1988) argued that there is not a clear indication of risk experience in the framework and that SARF centers on the information processing by individuals and evidently neglects the processes of social aggregation. The author also pointed out that the evaluation of effects of social responses are absent in SARF. Moreover, the definition of risk amplification is still vague as SARF does not explicitly specify the baseline risk against which amplification can be measured. These issues prevent SARF from being implemented in a simulation in any direct way. This paper shows how this can be done, with a more specific model and an agent-based, computational implementation. The model formalizes social amplification of risk through integrating direct experience, social interaction, media, and a recall process into the underlying dynamics of public risk perception. It demonstrates how risk perception evolves as result of interactions of various factors and yields valuable insights on the mechanism of risk amplification.

Calibration of the model using empirical data from a public survey about a specific product crisis practically means that the model is not general: its structure is generic, but calibrated parameter values are completely specific to the survey context. On the one hand, the model is generic in the sense that its construction draws on general knowledge of social risk amplification and product recall. It synthesizes factors that are identified as significant drivers of risk perception and incorporates interactions between different actors that are seen as influential in past empirical work. On the other hand, the calibrated model is highly context specific as the weights in the decision rule are completely dependent on the empirical phenomenon presented in the survey. Essentially, the recall model is partly calibrated, since no attempt is made to calibrate global input parameters (e.g. the objective risk level). Nonetheless, it is evident that the output traces generated by the model quite probably vary from one risk event or recall crisis to another as the space of parameters are restricted by the empirical evidence. As a consequence, the test of model validity requires replicating the model with empirical data sets in different contexts and in different places, as well as comparing results from various cases. This merits investigation in future research.

The main contribution of our paper is the generic model and the process of calibration. The conceptual model combines research emerging from both social risk amplification and product recall and brings diverse types of risk information into a single model. Therefore, it should be helpful in gaining a more comprehensive insight into the formation of risk perception in a product recall crisis. Furthermore, the generic model provides a basis for developing models of this kind by adding or taking away parameters. The calibration exercise carried out in this study helps identify the potential space of parameters for which data is readily available and improve the precision of model predictions. Calibration can, as Fagiolo et al. (2007) have suggested, 'bring modeling more closely in line with the empirical evidence and thereby reduce the dimensionality of a model'. Another contribution is that sensitivity analysis provides important indications on which model parameters have the biggest effects on outcome variables. Based on results displayed in this paper, the initial condition, contamination level, contamination duration, and recreancy variation are the parameters that peak risk amplification is very sensitive to. More importantly, sensitivity analysis help specify the parameters where data collection efforts need to be concentrated and which need most managerial attention due to their potential to cause large uncertainties in the outcome critical to an organization.

The main practical value of the study is to show policy makers and risk managers who need to deal with public risk responses how to reason about the problem of how such responses are formed. Every situation will be different, so it is unreasonable to claim any single model as being definitive. But the process of separating individuals' responses into a risk discovery step and a recreancy judgment step is an important one. Recognizing how interaction with social neighbors, broadcast media and producer announcements is also an important step. And thinking about the role of the media as leaders or followers of public responses has been shown to make an important difference. But, perhaps most significantly, the model helps managers reason about the effects of early or late product recalls, and help them deal with the dilemma that product recalls can be both harmful and beneficial to a producer's reputation. 
Liu, Busby, and Onggo

A basic limitation of our model is the use of very simple linear belief updating rules. In our model factors contributing to risk perception are combined using simple linear equations mainly due to the absence of firm evidence on the form in which they account for the belief updating process. Both Axelrod (1997) and Macal and North (2005) have showed that applying simple rules to agent-based models can result in emergent and complex behaviors and reveal important insights on what is being studied. Simulation results in this paper also show that our model is able to produce significant risk behavior and help understand social risk amplification more precisely. In addition, Axelrod (1997) pointed out that a very simple model allows making potentially interesting extensions to the present model. But there is scope to explore many other processes of interaction between agents and their social neighbors, the broadcast media, and producers dealing with product recalls.

\section{REFERENCES}

Axelrod, R. 1997. "The Dissemination of Culture: A Model with Local Convergence and Global Polarization”. The Journal of Conflict Resolution vol. 41, pp. 203-226.

Binder, A. R., D. A. Scheufele, D. Brossard, and A. C. Gunther. 2011. "Interpersonal Amplification of Risk? Citizen Discussions and Their Impact on Perceptions of Risks and Benefits of A Biological Research Facility”. Risk Analysis vol. 31, pp. 324-334.

Burns, W. J., and P. Slovic. 2007. "The Diffusion of Fear: Modeling Community Response to A Terrorist Strike”. The Journal of Defense Modeling and Simulation: Applications, Methodology, Technology vol. 4, pp. 298-317.

Busby, J. S., and S. Onggo. 2013. "Managing the Social Amplification of Risk: A Simulation of Interacting Actors”. Journal of the Operational Research Society vol. 64, pp. 638-653.

Busby, J. S., B. S. S. Onggo, and Y. Liu. 2016. “Agent-Based Computational Modelling of Social Risk Responses”. European Journal of Operational Research vol. 251, pp. 1029-1042.

Fagiolo, G., A. Moneta, and P. Windrum. 2007. "A Critical Guide to Empirical Validation of AgentBased Models in Economics: Methodologies, Procedures, and Open Problem”. Computational Economics vol. 30, pp. 195-226.

Freudenburg, W. R. 1993. "Risk and Recreancy: Weber, the Division of Labor, and the Rationality of Risk Perceptions”. Social Forces vol. 71, pp. 909-932.

Freudenburg, W. R. 2003. "Institutional Failure and the Organizational Amplification of Risk: The Need for A Closer Look”. In The Social Amplification of Risk, edited by N. Pidgeon , R. E. Kasperson, and P. Slovic, pp. 102-120. Cambridge, Cambridge University Press.

Kasperson, R. E. 2012. “The Social Amplification of Risk and Low-Level Radiation”. Bulletin of the Atomic Scientists vol. 68, pp. 59-66.

Kasperson, R. E., O. Renn, P. Slovic, H. S. Brown, J. Emel, R. Goble, J. X. Kasperson, and S. Ratick. 1988. "The Social Amplification of Risk: A Conceptual Framework”. Risk Analysis vol. 8, pp. 177187.

Macal, C. M., and M. J. North. 2005. “Tutorial on Agent-Based Modeling and Simulation”. In Proceedings of the 2005 Winter Simulation Conference, edited by M. E. Kuhl, N. M. Steiger, F. B. Armstrong, and J. A. Joines, pp. 2-15. Piscataway, New Jersey, Institute of Electrical and Electronics Engineers, Inc.

Magno, F. 2012. "Managing Product Recalls: The Effects of Time, Responsible vs. Opportunistic Recall Management and Blame on Consumers’ Attitudes”. Procedia-Social and Behavioral Sciences vol. 58, pp. 1309-1315.

McComas, K. A. 2003. "Public Meetings and Risk Amplification: A Longitudinal Study”. Risk Analysis vol. 23, pp. 1257-1270. 
Mitchell, V. W. 1995. “Organizational Risk Perception and Reduction: A Literature Review”. British Journal of Management vol. 6, pp. 115-133.

Moss, S., and B. Edmonds. 2005. "Sociology and Simulation: Statistical and Qualitative CrossValidation”. American Journal of Sociology vol. 110, pp. 1095-1131.

Muter, B. A., M. L. Gore, and S. J. Riley. 2013. “Social Contagion of Risk Perceptions in Environmental Management Networks”. Risk Analysis vol. 33, pp. 1489-1499.

Onggo, B. S., J. Busby, and Y. Liu. 2014. "Using Agent-Based Simulation to Analyse the Effect of Broadcast and Narrowcast on Public Perception: A Case in Social Risk Amplification”. In Proceedings of the 2014 Winter Simulation Conference, edited by A. Tolk, S. Y. Diallo, I. O. Ryzhov, L. Yilmaz, S. Buckley, and J. A. Miller, pp. 322-333. Piscataway, New Jersey, Institute of Electrical and Electronics Engineers, Inc.

Rip, A. 1988. “Should Social Amplification of Risk Be Counteracted?” Risk Analysis vol. 8, pp. 193-197.

Rogers, G. O. 1997. “The Dynamics of Risk Perception: How Does Perceived Risk Respond to Risk Events?” Risk Analysis vol. 17, pp. 745-757.

Scherer, C. W., and H. Cho. 2003. “A Social Network Contagion Theory of Risk Perception”. Risk Analysis vol. 23, pp. 261-267.

Sjöberg, L. 2000. “Factors in Risk Perception”. Risk Analysis vol. 20, pp. 1-12.

Smith, H. B., K. L. Dickinson, P. Champ, and N. Flores. 2013. "Social Amplification of Wildfire Risk: The Role of Social Interactions and Information Sources”. Risk Analysis vol. 33, pp. 800-817.

Watts, D. J., and S. H. Strogatz. 1998. “Collective Dynamics of 'Small-World' Networks”. Nature vol. 393, pp. 440-442.

\section{AUTHOR BIOGRAPHIES}

YUN LIU is a $\mathrm{PhD}$ student at the Department of Management Science at Lancaster University Management School, Lancaster, United Kingdom. Her research deals with risk management. He email address is y.liu30@lancaster.ac.uk.

JERRY BUSBY is Senior Lecturer (Associate Professor) at the Department of Management Science at Lancaster University Management School, Lancaster, United Kingdom. His research interests are in the analysis and management of risk. His email address is j.s.busby@lancaster.ac.uk.

BHAKTI STEPHAN ONGGO is Lecturer (Assistant Professor) at the Department of Management Science at Lancaster University Management School, Lancaster, United Kingdom. His research interests are in the areas of simulation methodology, simulation technology and simulation modeling in business and management. His email address is s.onggo@lancaster.ac.uk. 